\title{
BIOÉTICA Y BIOTECNOLOGÍA: LO HUMANO ENTRE DOS PARADIGMAS
}

\author{
Fermín Roland Schramm* \\ Miguel Kottow Lang**
}

\begin{abstract}
Resumen : Este trabajo intenta analizar algunos aspectos interaccionales entre biotecnología y bioética, así como esclarecer el rol paradigmático de estas disciplinas en la visión antropológica del hombre contemporáneo y de futuras generaciones humanas. Sugerimos que la biotecnología, sobre todo a través de la intervención genética, cuestiona ciertas constantes antropológicas y debe ser acompañada por una reflexión bioética dotada de perspectivas esclarecedoras y propositivas, que incluyan la regulación social de los proyectos biotecnológicos, la ponderación de beneficios y riesgos en sus aspectos de sustentabilidad social y ética, la propuesta de mecanismos decisionales vinculantes, el control prudente de las propias actividades regulatorias y la promoción de la equidad en el acceso a los bienes generados por la biotecnociencia.

Palabras clave: Bioética, biotecnociencia, biotecnología, sustentabilidad.
\end{abstract}

\section{BIOETHICS AND BIOTECHNOLOGY: THE HUMAN BETWEEN TWO PARADIGMS}

Abstract : This paper intends to analyse some of the interactions that occur between biotechnology and bioethics, considering that both disciplines have a truly paradigmatic character that influences the anthropologic views of modern man and future human generations. We suggest that biotechnology, especially in the form of genetic interventions, questions certain anthropologic constants and must therefore be subjected to descriptive and prescriptive bioethical reflection. Benefit/risk ratios must be analyzed from the social and the ethical points of view, effective decisional mechanisms need to be suggested, and prudential regulation will have to be envisioned as well as the promotion of equity in the access to biotechnologic benefits.

Keywords: bioethics, biotechnology, biotechnoscience, sustainability.

\section{BIOÉTICA E BIOTECNOLOGIA: O HUMANO ENTRE DOIS PARADIGMAS}

Resumo: Este trabalho procura analisar alguns aspectos de interação entre biotecnologia e bioética, assim como esclarecer o elenco paradigmático destas disciplinas na visão antropológica do homem contemporâneo e de futuras gerações. Entendemos que a biotecnologia, sobretudo através da intervenção genética, questiona certas constantes antropológicas e deve ser acompanhada de reflexão bioética dotada de perspectivas esclarecedoras e propositivas que incluam a regulação social dos projetos biotecnológicos, a ponderação de riscos e benefícios nos aspectos de sustentabilidade social e ética, a proposta de mecanismos decisionais vinculantes, $o$ controle prudente das próprias atividades reguladoras e a promoção da equidade no acesso aos benefícios gerados pela biotecnociência. Palavras chaves: Bioética, biotecnociência, biotecnologia, sustentabilidade.

* Ph.D. Investigador Asociado de la Escuela Nacional de Salud Píblica de la Fundación Oswaldo Cruz de Río de Janeiro, Brasil. Profesor Visitante de la Escuela de Salud Pública de la Facultad de Medicina de la Universidad de Chile.

Correspondencia: Escola Nacional de Saúde Pública da Fundaçao Oswaldo Cruz. Rua Leopoldo Bulhoes 1480, Sala 921. Manguinhos, Río de Janeiro, RJ, 21041-210, Brasil.

** Magíster en Sociología. Doctor en Medicina. Profesor Titular de la Universidad de Chile, Facultad de Medicina y Facultad de Filosofía y Humanidades.

Correspondencia:guarvie@ctcreuna.cl 


\section{BIOÉTICA Y BIOTECNOLOGÍA: LO HUMANO ENTRE DOS PARADIGMAS ${ }^{\mathrm{i}}$}

\section{Introducción}

Las vertiginosas transformaciones de la tecnociencia y su impacto sobre las visiones de mundo contemporáneamente vigentes, así como las crecientes exigencias a la reflexión que atañe a estas transformaciones, son todos factores que han acelerado un discurso ético cuyos conceptos y términos no han logrado decantar en un juego de lenguaje de común aceptación sino, al contrario, llevan a la utilización de significantes cuyas lecturas no son coincidentes para los diversos interlocutores. De allí que las éticas aplicadas se ven obligadas de definir los términos de su argumentación y buscar la univocidad de algunas figuras retóricas cuya riqueza de connotaciones dificultan los entendimientos.

Estas dificultades son especialmente notorias en disciplinas emergentes como la bioética y la tecnociencia aplicada a los fenómenos vitales. El presente trabajo intenta dirimir algunos aspectos confrontacionales entre bioética y biotecnología, así como esclarecer el rol paradigmático que puedan estar jugando en la visión antropológica del hombre contemporáneo y, no menos, de futuras generaciones humanas, lo cual requiere desde el inicio un intento por clarificar algunos conceptos fundamentales.

Por ello, antes de hablar sobre la nueva condición moral humana frente a lo que llamamos vigencia del paradigma biotecnológico, es necesario especificar el sentido en que se utilizarán los términos "paradigma", "biotecnología"y "bioética", ante todo por la sospecha razonable de que no tienen un significado consensual.

i Trabajo realizado durante la permanencia postdoctoral de Fermín Roland Schramm en la Universidad de Chile.

\section{Definiendo los términos "bioética", "paradigma" y "biotecnología"}

La bioética, por de pronto, ha tenido definiciones muy dispares que fluctúan desde homologarla con la ética médica, hasta darle funciones y responsabilidades de una ética global que abarque todas las actividades y todos los intereses que el ser humano pueda imaginar con relación a la sobrevivencia de la humanidad y del mundo (1). Dada la "diversidad de prácticas y de discursos llamados 'bioéticos' no existe un concepto simple y absolutamente unívoco" (2). Entendemos por bioética la reflexión ética en torno a actos humanos que alteran de forma definitiva los procesos vitales - en tanto irreversibles (3). Esta definición es lo bastante amplia y suficientemente precisa porque no se restringe al mero ámbito biomédico, sino que engloba la moralidad de las prácticas biotecnológicas y de la ecología, delimitando, al mismo tiempo, aquellos aspectos de la vida que son pertinentes para el análisis moral.

La noción de paradigma ha tenido, a su vez, una variedad de significados. T. Kuhn (4)le da un sentido técnico de matriz disciplinaria o sistema de hipótesis, métodos y protocolos de investigación, compartidos por los profesionales de un determinado campo disciplinario; en el sentido más tradicional, paradigma corresponde a las acepciones de la palabra griega paradeigma en tanto "modelo de referencia" a ser imitado (Platón, Timeo, 29b, 48e) o de mero "ejemplo" (Aristóteles, Analítica Priora, II, 24, 68b). Todos estos sentidos son legítimos pues cada uno indica un padrón de referencia para alguna función y, por ende, todos respetan el sentido principal de la palabra. Tal vez sea conveniente, sin embargo, reservar el carácter de paradigmáticos a aquellos acontecimientos que signifiquen una transformación cultural, sea específica para el campo en el cual se da el paradigma, sea en una visión más general de los valores y las visiones prevalentes. Charles Darwin, por ejemplo, provocó un cambio paradigmático 
amplio en la biología, en la antropología, en la teología y en la filosofía, en tanto que el descubrimiento de la insulina significó un impacto sin duda fuerte pero enfocado al campo de la endocrinología médica.

El término "biotecnología" viene siendo utilizado desde que se produjera la simbiosis entre avances científicos, capacidades técnicas y su aplicación a la modificación y la generación de fenómenos vitales, así como a la creación de seres vivos a partir de métodos de laboratorio. La biotecnología encubre el hecho previo de la amalgama entre ciencia y técnica -tecnociencia- que se produce a partir del siglo XVII y que tiene por característica que el conocimiento científico sirve en forma directa e inmediata al desarrollo técnico el cual, a su vez, nutre a la ciencia con instrumentos de investigación y observación cada vez más sofisticados; con lo cual se vuelven obsoletas dos cuestiones que también interesarán a la biotecnología: 1) Desaparece la brecha entre investigación pura y aplicación, y 2) La ciencia no puede declararse inmune a la valoración ética, ya que su producción de inmediato adquiere presencia social y, con ello, peso valórico. La biotecnología, que es una tecnociencia aplicada a lo vivo y que, en rigor, debiera llamarse "biotecnociencia" (5) comparte estas dos características de no distinguir entre conocimiento y aplicación, y de llevar por ende en todas sus etapas una fuerte carga valórica. La simbiosis entre conocimiento y aplicación adquiere nuevas dimensiones si se considera que los altos costos del desarrollo tecnocientífico gatillan su traslado al ámbito de la empresa privada, lo cual implica una fuerte presencia de valores comerciales y el primado de intereses monetarios por sobre los académicos.

Si bien el término "biotecnología" contiene dos errores -calla el aspecto de la ciencia y habla de un logos que no corresponde a la técnica sino a la reflexión sobre ella-, ha tenido tal difusión que será aquí utilizado en el sentido de ser una práctica tecnocientífica ejercida sobre lo vivo, en una homología algo espuria entre biotecnología y biotecnociencia.

\section{Las tres etapas de la biotecnología}

El término biotecnología (en el sentido de biotécnica) es generalmente utilizado en el sentido restringido de aplicación de la ingeniería genética y de las técnicas del ADN recombinante a los sistemas vivos. Esta utilización es prima facie correcta, visto que lo públicamente visible, por ejemplo en los debates sobre Organismos Genéticamente Modificados (OGM), son sobre todo los productos de la biotécnica transgénica. Estos debates también forman parte de la agenda de ambientalistas, defensores de consumidores, y organizaciones no gubernamentales (ONG), preocupados de los modos de producción de OGM y de sus posibles o probables efectos sobre la salud y el bienestar de las personas, la economía, las relaciones entre consumidores y productores, la transformación del medio ambiente a medio y largo plazo. La utilización reduccionista de la palabra "biotecnología" puede ser bastante problemática en la medida que ignora esta diversidad de intereses comprometidos y las reflexiones que ella desencadena, dejando sin efecto lo que Thompson ha denominado el principio de la soberanía del consumidor (6).

Hablar de biotecnología, sobre todo en un sentido crítico, suscita de inmediato la observación que nada radicalmente nuevo estaría ocurriendo, puesto que el hombre siempre ha influido sobre los seres vivos y sobre la naturaleza. De allí que sea necesario distinguir al menos tres etapas de la biotecnología, la primera siendo efectivamente muy antigua y coincidiendo con la revolución agrícola que domesticó a las plantas comestibles y a diversas especies animales. Los primeros agricultores dirigían la selección natural, cuidando de especies que les parecían útiles y descuidando las variedades o especies inconvenientes. Bien mirado, era ésta una bioprototécnica, en la cual los procesos naturales eran modificados dentro de sus 
capacidades autopoiéticas, es decir, una cepa vegetal o una variedad animal podían ser artificialmente cultivados en tanto ello no sobrepasara su capacidad natural de adaptación, lo cual impedía que se produjeran grandes desajustes ecológicos.

La segunda etapa, ejemplificada por la crisis contemporánea de la "vaca loca", implica una interferencia biotecnocientífica en una especie animal que excede su tolerancia biológica, prueba de lo cual es que la alimentación artificialmente carnívora que se dio al ganado vacuno produjo en un número importante de especímenes una enfermedad mortal, la Encefalopatía Espongiforme Bovina (EEB). Sería interesante conjeturar sobre la importancia que este accidente pecuario habría tenido si la carne infectada no hubiese provocado en el ser humano la también letal Enfermedad de Creuzfeldt-Jacobs.

Muy probablemente el mal de la vaca loca es consecuencia de la decisión pragmática de algunos criadores que escogieron alimentar su ganado con harinas animales disponibles en el mercado, transformando sus rebaños herbívoros en omnívoros. Hubo por ende un error de ellos y quizás una falta de competencia pues -se puede argumentar- ¡deberían saber que las vacas no comen carne! Pero, también es legítimo preguntar: antes de que los industriales comercializaran tales harinas, ¿fueron hechas las investigaciones necesarias para poder prever la probabilidad de riesgos para el bienestar animal y la salud humana, incluyendo posibles impactos sobre el medio ambiente? Es decir, ¿Fueron respetadas las normas de seguridad? ¿Se informó a los criadores sobre el contenido del nuevo producto alimentario, a fin de permitirles decidir si comprarlo o no? Las declaraciones de diversas instancias sociales y de varios gobiernos reconocen que hubo mucha información encubierta, lo cual permitió la predominancia de intereses pecuniarios por sobre el resguardo sanitario de seres vivos.

La EEB muestra cuán fácil es, por medio de acciones técnicas aparentemente inocuas como la de mudar los hábitos alimentarios de animales, llegar a una situación epidémica con graves consecuencias para la salud y el bienestar no sólo del ganado, fuente de lucro para sus criadores, sino también de riesgo letal para poblaciones humanas. Y eso tiene, por supuesto, implicaciones bioéticas si aceptamos nuestro presupuesto de que esta disciplina se refiere a las prácticas humanas que pueden alterar irreversiblemente los procesos vitales -que a su vez son irreversibles-, dañando a seres humanos y animales, así como al medio ambiente.

En términos más técnicos, el caso de la EEB es un claro ejemplo de cómo la acción humana puede alterar la homeostasis de un sistema vivo, volviéndolo incompetente para "regenerarse desde adentro", de "transformar la materia/energía en un proceso interno de automanutención y autogeneración" (7, p.31), o de mantener sus defensas frente a agresiones ambientales -virales en la EEB. De hecho, esta autoorganización es importante para que cualquier sistema autopoiético se mantenga vivo, lo cual sólo puede lograrse si es autónomo y en la medida que pueda reconocer su entorno como propio o resistir eficazmente lo ajeno. Por ello se puede considerar que hay codeterminación entre un sistema vivo y su entorno o ambiente, visto que "[un sistema autopoiético] depende de su entorno físicoquímico para su conservación como entidad autónoma, de lo contrario se disolvería y reintegraría a su entorno" (7, p 59).

Hay diferencias obvias entre la relativa inocuidad de la bioprototécnica y la biotecnología contemporánea aplicada al ganado vacuno, que altera la biología de una especie al punto de romper la cadena nutricia natural con la destrucción de la especie interferida -los vacunos- y de la especie que se alimenta de ella, los seres humanos. En esta segunda fase de la biotecnología hay debilitamiento y aniquilación de sistemas autopoiéticos, así como reacciones en cadena absolutamente inesperadas. Este vuelco sorpresivo es característico de la tecnociencia 
$\mathrm{y}$, por ende, de la biotecnociencia compleja, donde la instrumentación no logra prever todos los efectos posibles que puede desencadenar, como ya lo reclamara Hans Jonas (8).

En recientes lustros se ha producido un nuevo salto cualitativo en las prácticas biotecnológicas, inaugurando lo que podría denominarse su tercera fase, cuyo sustrato es la genética y cuyo producto más obvio es la transgenia o el traslado instrumental de material genético entre diversas especies. La característica más relevante de las técnicas transgénicas es la creación de nuevos genomas, vale decir, de nuevas especies. El conocimiento genético actual dista mucho de ser tan completo como para predecir todas las transformaciones que la transgenia pudiese producir en las nuevas especies y eso significa, extrapolando del caso de las "vacas locas", que el potencial de sorpresas y de efectos deletéreos inesperados es inconmensurable -literalmente no se puede medir.

En este paradigma biotecnológico que hemos denominado fase tres, aparece un fenómeno inédito, pues el hombre ha ganado un enorme ascendiente sobre los seres vivos y su entorno, pero al mismo tiempo ha traspasado el límite, hasta ahora infranqueable, de dirigir su potencial biotecnológico hacia sí mismo. La genética ha posibilitado que el ser humano altere a todos los seres vivos, incluyendo a la especie humana, en forma irreversible e inconmensurable. Salta a la vista que la razón pragmática posibilita cambios antropológicos profundos que la bioética debe analizar en forma acuciosa y oportuna.

\section{El quehacer humano entre paradigma biotecnológico y paradigma bioético}

El paradigma biotecnológico indica un nuevo campo de estudios y prácticas resultantes de la aplicación de la tecnociencia moderna al ámbito de la vida y de la salud. Este campo resulta de la alianza entre: (a) el saber, tanto teórico como práctico de la tecnociencia o, mejor dicho, "la determinación pragmática de la teoría" $(8$, p.271) desarrollada sobre todo por la física experimental y la ingeniería; (b) el saber, también teórico y práctico, desarrollado por especialistas de las ciencias de la vida tales como biólogos y médicos; y (c) el saber, igualmente teórico y práctico, desarrollado por los profesionales de la informática. Es la sinergia de estas tres áreas epistemológicas y técnicas en el campo del saber que indicamos aquí como vigencia del paradigma biotecnológico.

La emergencia del nuevo paradigma biotecnológico hace patente no solamente que existe un "uso práctico de la teoría" (que caracteriza a la tecnociencia desde su inicio) sino también que "el fenómeno mismo de la vida niega las fronteras que suelen separar nuestras disciplinas y campos de trabajo entre sí” (8, p.11).

La re-programación de un sistema vivo puede tener dos objetivos: (1) un objetivo predictivo-preventivo, consistente en evitar alguna característica considerada negativa, responsable de enfermedades, disfunciones o molestias; (2) un objetivo meliorativo o perfectivo, en el sentido de fortalecer sus funciones, reforzar una característica existente o propiciar una nueva, considerada deseable. Pero todo eso ocurre bajo condiciones de incertidumbre y sobre todo con riesgos difícilmente ponderables, lo cual es tanto más grave si la intervención tiene fines meliorativos, o sea, estimables como superfluos, que si se hace con objetivos terapéuticos. En todo caso, es posible considerar que la vida se convierte en "un experimento con apuestas y riesgos crecientes, que la libertad del hombre, preñada de peligros, puede llevar tanto a la catástrofe como al éxito" (8, p.10).

Posiblemente fue Hans Jonas el más preclaro de los observadores críticos del paradigma biotecnológico y de las cuestiones morales inéditas que implica. Pensando en una nueva ética, planteó el principio de responsabilidad óntica, según el cual lo principal era asegurar que el ser humano siga existiendo a futuro (9). Algo más tarde, pero 
también enfocado a los problemas de la ética aplicada en el campo biotécnico, G. Hottois acuñó el término de "paradigma bioético" (10).

La potencia instrumental y la capacidad práctica de la biotecnociencia son profundamente inquietantes, al mismo tiempo que acusan una fuerte resistencia de la razón pragmática frente a consideraciones éticas. Imperativo tecnológico fue el término con que Jonas caracterizó esta dinámica del saber hacer que no se deja frenar por temores o preocupaciones de orden valórico. Si todo lo que se puede hacer se hará, nace la pregunta acaso la reflexión bioética tenga alguna posibilidad de influir sobre los acontecimientos. El propio Jonas parece escéptico frente a esa posibilidad cuando afirma que "el uso de la teoría científica es imparable, pues parar su uso querría decir parar la teoría misma, y no nos está permitido detener la marcha del saber: si no a causa de las ganancias que el saber proporciona, si en cualquier caso en atención a los costos que supondría esa detención" y porque "tanto la honradez como la lógica nos vedan el camino de vuelta a la posición clásica"; en suma, porque "la teoría misma se ha convertido en un proceso que (...) comporta su propio uso práctico, y no se la puede 'poseer' de otra manera" (8, p.276). Además, si eso vale para la mera teoría (como podría ser el análisis filosófico de lo vivo) debe valer a fortiori para la tecnociencia y las biotecnologias modernas en las cuales no sólo "la teoría está en función del uso en la misma medida en que el uso está en función de la teoría".

Por ende, tecnociencia y biotecnologías traen para el ser humano un "nuevo reino de la necesidad" en substitución de las necesidades que ellas pretendían satisfacer, razón por la cual Jonas concluye que "si equiparamos el reino de la necesidad a la 'caverna' de Platón, observamos que la teoría científica no nos saca de ella, ni su aplicación práctica es una vuelta a la misma: nunca la ha abandonado" (8, p.277). Mas, ¿qué se puede hacer en la caverna de Platón? ¿Qué rol puede jugar la bioética considerando la situación denunciada por Jonas?

\section{El quehacer de la bioética en la caverna de Platón}

Si consideramos que la bioética es una ética aplicada, le corresponde una doble tarea: una descriptiva y de esclarecimiento, la otra normativa y de propuesta decisional. La primera se acerca a la tarea que Platón atribuye al filósofo al retornar a la caverna, es decir, la tarea de la bioética sería analizar los hechos y el proceso biotecnológico del modo más imparcial posible, ponderando a la luz de la razón -que es la heredera del antiguo logos- los argumentos en pro y en contra del cultivo de la biotecnociencia en general y de cada caso específico de su aplicación.

El papel de la teoría es evitar cualquier tipo de fundamentalismo en el análisis moral y sugerir, por ende, que las decisiones a tomar sean acuciosa y ponderadamente estudiadas. En particular, la teoría permite que las biotecnologías sean miradas no solamente en su potencial de amenazas hacia "el futuro total" (8, p.323), sino también en sus posibles y probables beneficios para los seres vivos $\mathrm{y}$, naturalmente, los seres humanos. Es discutible la idea de Jonas, in dubio pro malo, pues puede tener por consecuencia la inacción y en ese caso habrá que justificar, con buenos argumentos, por qué el no actuar implica menos riesgos que el actuar, visto que la falta de beneficios también puede ser un efecto negativo. En otras palabras, admitiendo que tanto la acción como la inacción pueden ser comportamientos de riesgo, la bioética necesita de mucha competencia para ponderar riesgos y beneficios, aplicando el principio de prudencia a su vez prudentemente, aun ante la tentación de controlar y restringir los proyectos biotecnológicos. Pero en aquellas situaciones, cada vez más frecuentes, en que la ponderación de beneficios vs. riesgos sea demasiado incierta, habrá que tener en cuenta el escepticismo de Jonas, quien llamaba a la frugalidad en situaciones de mucha incertidumbre, tanto más si se considera que la 
evolución de la tecnociencia ha sido hacia mayor complejidad y un aumento tanto de los riesgos efectivos (11), como del temor a estos riesgos (12). Frugalidad y prudencia deben estar tanto más presentes en la fase tres de la biotecnociencia, donde los efectos de la intervención humana en la vida son difíciles de predecir e irreversibles.

La biotecnociencia influye sobre la sociedad en general y altera las relaciones culturales que en su seno se despliegan. Debido a estas vastas transformaciones la biotecnociencia es indiscutiblemente un nuevo paradigma epistemológico y pragmático. El debate en torno a los logros, los valores y los riesgos de la biotecnociencia no pueden reducirse a una discusión en el cenáculo de expertos, siendo función de la bioética lograr que la labor de esclarecimiento, la iluminación de la caverna o la mostración del camino para salir de ella, han de hacerse en forma ampliamente comunicativa, por cuanto las resoluciones que emanen de la reflexión bioética deberán reproducir el sentir y el pensar de la sociedad in toto e, idealmente de acuerdo con Habermas, de cada uno de sus miembros (13). Más allá de la sustentabilidad económica que suele ser analizada para empresas tecnocientíficas de alto costo, se trata de plantear y dirimir la sustentabilidad social, vale decir, el espectro y la magnitud de los riesgos que la sociedad está dispuesta a asumir en vista de los beneficios a esperar.

El esclarecimiento ciudadano genera recomendaciones y resoluciones para la biotecnociencia; esta normativa tiene por objetivo evitar abusos de poder de empresas biotecnológicas, instituciones tecnoburocráticas y del mercado. Se puede llamar a este primer plano de interacción de la bioética con la biotecnociencia una condición necesaria para el control de la actividad biotecnológica.

También de Jonas proviene el escepticismo de que la biotecnología sea regulable a través de un discurso bioético, por muy amplia que sea su base social. Pero, plantea el mismo autor, la magnitud de los acontecimientos impide entregarse al desaliento, pues "en los instantes de la decisión, cuando todo nuestro ser se pone en juego, sentimos como si estuviésemos actuando ante la mirada de la eternidad " (8, p.309) . E1 instante de la decisión ya no es solamente el de la acción individual a corto plazo sino, ante todo, el momento del "género humano en su obrar social global" visto que "[n]inguna escondida moralidad en la existencia privada, sólo la acción pública y colectiva en pos de efectos que se produzcan aquí, puede conjurar ese peligro, y no sabemos qué alianzas con el mal tendrá el bien para impedir lo todavía peor, incluso lo absolutamente inadmisible" (8, p.322-3).

Este lenguaje, tal vez un poco rapsódico, requiere de la bioética que lleve el debate público más allá del mero esclarecimiento, proponiendo mecanismos decisionales que sean vinculantes para apoyar o frenar una determinada práctica biotecnológica. Este segundo plano operacional e interaccional constituye la condición suficiente para el control de la actividad biotecnológica.

El control de actividades riesgosas conlleva, a su vez, los riesgos del control. El control tecno-burocrático de la ciencia por el Estado, actualmente muy reducido en su gestión científica, debe evitar la propagación de políticas autoritarias e incluso dictatoriales, de tan triste memoria por sus nefastos efectos sobre el desarrollo de la ciencia. Por otra parte, el control ciudadano ha de ser esclarecido y respetuoso del multiculturalismo, a objeto de evitar que dogmatismos y fundamentalismos retrasen el progreso de ciencia y técnica en áreas de beneficio social y de mejor cobertura de necesidades primarias. Las actividades regulatorias deben, a su vez, ser cuidadosamente analizadas, con lo cual se establece un tercer plano de interacción entre bioética y biotecnociencia, caracterizado por el control del control.

Especial cuidado debe tener la reflexión bioética al evaluar la tendencia de la 
biotecnociencia a aumentar las brechas entre los que acceden a ella y quienes quedan marginados. Mientras más complejas y costosas, más privadas son las iniciativas tecnocientíficas y biotecnocientíficas, de manera que sus productos se vuelven artículos de lujo sólo accesibles a los pudientes en naciones desarrolladas y en desarrollo. Un aspecto perverso de esta distribución asimétrica es que los beneficios quedan en pocas manos en tanto que los riesgos invaden ámbitos mucho más amplios y producen deterioros de calidad de vida -medio ambiente poluto y tóxico, alimentos transgénicos de altos costos, congestiones urbanas- que afectan a toda la ciudadanía. Más allá de esta falta de ecuanimidad en la distribución de beneficios y daños/riesgos, la bioética ha de velar porque no se produzca el fenómeno inverso, de una contracción biotecnológica en áreas que desprotejan las necesidades de los más desposeídos. En este plano la bioética pregunta por la sustentabilidad ética de los proyectos - ecuanimidad en la distribución de beneficios y riesgos-, constituyéndose el control de la equidad de ciencia y técnica.

\section{Conclusiones}

Con la introducción a gran escala de las técnicas de intervención genética, se inicia una nueva era biotecnocientífica, con un potencial de efectos positivos pero también negativos, que sobrepasan los límites espaciotemporales hasta ahora conocidos. Ello genera por un lado la esperanza de obtener nuevos instrumentos técnicos para mejorar la adaptación del ser humano, y de los seres vivos en general, al medio ambiente, pero, por otra parte, abre también los temores de generar daños y riesgos de incalculable dimensión y que, por su irreversibilidad, tendrían efectos transmitidos a futuras generaciones.

Esta notable expansión de la razón instrumental debe acompañarse de una fortificación a nivel social de la ponderación valórica, lo cual asigna a la bioética la tarea de enriquecer y robustecer el discurso comunicativo. Esta tarea tiene un componente reflexivo fundamental, pero que sólo será eficaz si logra generar mecanismos de recomendación y de resolución que ayuden a regular las actividades biotecnocientíficas en respeto del bien común.

\section{Agradecimientos}

Este trabajo fue realizado con el apoyo del CNPq, entidad del Gobierno Brasileño que apoya el desarrollo científico y tecnológico. 


\section{Referencias}

1. Potter VR. An essay review of global responsibility in search of a new world ethics. Perspectives in Biology and Medicine 1994; 37: 546-50.

2. Hottois G. Bioéthique. In: Hottois G, Parizeau MH (eds). Les mots de la bioéthique. Bruxelles: De BoeckWesmael; 1993. p. 52.

3. Kottow M. Introducción a la bioética. Santiago de Chile: Universitaria; 1995.

4. Kuhn T. The structure of scientific revolutions. Chicago: The University of Chicago Press; 1962.

5. Schramm FR. Paradigma biotecnocientífico e paradigma bioético. In: Oda LM (ed). Biosafety of transgenic organisms in human health products. Rio de Janeiro: Fiocruz; 1996. pp. 109-27.

6. Thompson PB. Food biotechnology's challenge to cultural integrity and individual consent. Hastings Center Report 1997; 27: 34-8.

7. Varela F. El fenómeno de la vida. Santiago de Chile: Dolmen; 2000.

8. Jonas H. El principio vida: hacia una biología filosófica. Madrid: Trotta; 2000.

9. Jonas H. Das Prinzip Verantwortung. Frankfurt a. M.: Insel Verlag; 1979.

10. Hottois G. Le paradigme bioéthique: une éthique pour la technoscience. Bruxelles: De Boeck-Wesmael; 1990.

11. Beck U. Risikogesellschaft. Auf dem Weg in eine andere Moderne. Frankfurt a. M.: Suhrkamp Verlag; 1986.

12. Luhmann N. Ökologische Kommunikation. Opladen: Westdeutscher Verlag; 1986.

13. Habermas J. Moralbewusstsein und kommunikatives Handeln. Frankfurt a. M.: Suhrkamp Verlag; 1981. 